\title{
L'HOMME L'Homme
}

Revue française d'anthropologie

185-186 | 2008

L'anthropologue et le contemporain : autour de Marc Augé

\section{Acteurs locaux, acteurs globaux}

Les néopentecôtistes en Amérique latine

Jesus Garcia-Ruiz

\section{(2) OpenEdition}

\section{Journals}

Édition électronique

URL : http://journals.openedition.org/lhomme/24198

DOI : 10.4000//homme.24198

ISSN : 1953-8103

Éditeur

Éditions de l'EHESS

Édition imprimée

Date de publication : 1 janvier 2008

Pagination : 387-399

ISSN : 0439-4216

\section{Référence électronique}

Jesus Garcia-Ruiz, «Acteurs locaux, acteurs globaux », L'Homme [En ligne], 185-186 | 2008, mis en ligne le 01 janvier 2010, consulté le 19 avril 2019. URL : http://journals.openedition.org/

Ihomme/24198; DOI : 10.4000/lhomme.24198 


\section{Acteurs locaux, acteurs globaux \\ Les néopentecôtistes en Amérique latine}

Jesus Garcia-Ruiz

$\mathrm{C}$

ET ARTICLE porte sur certains des effets majeurs du processus de privatisation du religieux, tel qu'il est en particulier intervenu dans l'aire culturelle étudiée, à savoir l'Amérique latine, et sachant que ce qui se joue sur le terrain du religieux traduit en réalité (et accompagne) des mutations intervenant dans d'autres registres : autrement dit le religieux s'est privatisé en même temps que le téléphone, les services, voire l'État.

On est sortis, sur fond de mondialisation où l'on assiste à un transfert de logiques du global au religieux, d'une gestion parapublique de celui-ci pour entrer dans une gestion privée. Au titre des effets induits par cette privatisation, on s'intéressera ici aux interactions de réseaux en relation avec les modalités de fonctionnement des Églises néopentecôtistes. Émerge de ces interactions la figure centrale du pasteur, articulant de façon spécifique les dimensions locale, transnationale et globale. Grâce aux réseaux, cet acteur est en situation d'incorporer dans les dispositifs qu'il met en œuvre tous les apports extérieurs dont ces réseaux sont le vecteur. Mais au-delà de la figure du pasteur, c'est l'articulation même du local et du global ainsi que les redistributions qui en résultent qui seront concernées, les terrains retenus étant les différents pays d'Amérique latine (et plus particulièrement le Guatemala). En ce sens, notre recherche se situe dans l'approche des logiques et de la nature des acteurs - terrain que Marc Augé a analysé par le biais de la figure du prophète. Il s'agit, comme il l'a souligné, de processus de symbolisation dans lesquels "les moyens se substituent aux médiations »; il s'agit de processus de construction de

Depuis de nombreuses années, les conversations avec Marc Augé, les séminaires auxquels nous avons participé tous deux, notamment au Guatemala, et la lecture de ses ouvrages m'ont aidé à élaborer ma réflexion sur l'expansion du protestantisme évangélique en Amérique latine. 
"médiations fonctionnelles ", c'est-à-dire, "de relations qui font sens parce qu'elles sont symbolisées et instituées dans les consciences des croyantsacteurs sociaux ${ }^{1}$.

De tous les mouvements religieux qui se sont développés dès le début du $\mathrm{XX}^{\mathrm{e}}$ siècle en Amérique latine, le néopentecôtisme est celui qui a eu la progression la plus rapide et qui, dans un temps relativement court, a réussi à se positionner parmi les classes aisées des sociétés. À la différence du pentecôtisme qui est dualiste ${ }^{2}$ (Corps/Âme, Terre/Ciel, Église/Monde), prémillénariste (il attend la Seconde Venue du Christ et la fin de l'histoire), et induit une conception de la personne caractérisée par un déterminisme individualiste $^{3}$ (il est « la scène » de confrontation, de batailles quotidiennes entre les forces du Bien et du Mal), les néopentecôtistes, pour leur part, ont une conception tripartite de la personne: Esprit, Âme et Corps. Dans la lutte contre Satan, qui est un être spirituel, c'est l'esprit qui livre la bataille. L'âme c'est le «je», la nature psychologique, le centre de la personnalité.

Cette conception tripartite se prolonge par la priorité accordée à la trilogie Esprit / Ciel / Église et jette un discrédit sur la triade Terre / Histoire / Société. Il s'agit donc d'une vision beaucoup plus instrumentale et opératoire. Les néopentecôtistes opposent à l'eschatologie défaitiste des pentecôtistes, une eschatologie de victoire. Convaincus que Dieu leur a délégué son pouvoir, Son autorité, Sa souveraineté et Sa domination - Power Evangelism -, ils mènent des stratégies permanentes de "conquête des nations ». Cette vision de "victoire" correspond mieux à la nature des groupes sociaux parmi lesquels s'épanouit le néopentecôtisme: les classes moyennes et la bourgeoisie. Dans ce contexte, les néopentecôtistes interprètent les antagonismes sociaux comme une " menace » du statut social de ses adeptes et comme une attaque du démon vis-à-vis de l'ordre social "voulu par Dieu ", et qu'il faut donc défendre. C'est le prolongement de la vision du monde de la «théologie de la prospérité » analysé par Ricardo Mariano ${ }^{4}$.

Dans le cas du Guatemala, de nombreux néopentecôtistes appartiennent à la classe des «nouveaux riches» et/ou à des groupes professionnels qui ont réussi et auxquels cette " théologie de la prospérité » apporte justification et légitimité. Cela est à l'origine de la construction d'une nouvelle identité politique et d'une légitimité nouvelle dans l'exercice du pouvoir.

1. Voir Marc Augé, "La leçon des prophètes", postface à Jean-Pierre Dozon, La Cause des prophètes. Politique et religion en Afrique contemporaine, Paris, Le Seuil, 1995.

2. Voir Jesus Garcia-Ruiz, "La conception de la personne chez les pentecôtistes et néopentecôtistes : Millénarisme, fin de l'histoire et pouvoir ", Socio-anthropologie, 17/18, 2006 : 151-186.

3. Voir Juan Sepúlveda, "La visión del hombre en el pentecostalismo chileno ", in Divers Ecce Homo, Santiago de Chile, $1983: 87$.

4. «Os neopentecostais e a Teologia da Prosperidade», Novos Estudos, 1996, 44 : 22-44, CEBRAP, Sao Paulo. 
L'appartenance à l'Église est la garantie d'incarner "l'avant-garde d'une guerre sainte contre les diverses formes de cancer moral " puisque "l'Église locale est une armée organisée, équipée pour se battre, toujours prête à anéantir l'ennemi ». L'eschatologie postmillénariste, propre aux néopentecôtistes, fait penser le monde comme un espace de reconquête, de concurrence et de compétition. Il ne s'agit pas d'un lieu de "pèlerinage et de transit" comme pour les pentecôtistes, c'est un lieu pour l'exercice du pouvoir. Le pentecôtisme reste, malgré ses logiques issues de la modernité, une institution hiérarchique proche des formes d'autorité et de gestion dérivées du fonds institutionnel du protestantisme historique. En revanche, le néopentecôtisme, issu de la globalisation, transforme en profondeur les registres d'autorité et les modalités de gestion : le pasteur est leader-propriétaire univoque de l'institution, qui est un bien familial privé et géré financièrement comme tel.

En fait, les processus massifs de conversion dont l'Amérique latine est le théâtre, mettent en scène des logiques de recomposition identitaire qui sont, en même temps, des processus de dé-historisation/ré-historisation des consciences à partir des représentations mobilisées d'un univers symbolique "importé » et historiquement situé. Il s'agit de valeurs et de pratiques issues du protestantisme nord-américain, et qui sont réappropriées par les acteurs locaux formés aux États-Unis. Ce contexte est à l'origine de la réarticulation, par le religieux, des relations individu/communauté et d'une nouvelle logique dans les rapports communauté / action politique, ce qui transforme la nature des relations du politique au religieux. Dans cette logique, le pasteur devient un acteur direct et indirect fondamental.

\section{Le néopentecôtisme et ses logiques}

La montée en puissance progressive du néopentecôtisme constitue un remarquable indicateur des phénomènes de dérégulation des systèmes traditionnels en Amérique latine tant au niveau culturel que religieux ou institutionnel. Elle représente simultanément une modalité privilégiée de reconstruction étroitement finalisée du social. On soulignera qu'à la différence du système catholique traditionnel, ce qui se met en place vise également à exercer un contrôle sur les activités individuelles, une maîtrise que le catholicisme, qui prétendait pourtant embrasser la totalité du social, n'était pas parvenu à réaliser.

Les sociétés latino-américaines ont été historiquement structurées suivant la logique baroque d'une organisation par le religieux de la totalité du social, axée sur une territorialisation définie par le cercle paroissial. Cette logique a connu, depuis la conquête de l'Amérique latine jusqu'à nos jours, plusieurs 
ajustements, passant de la figure de l'arrachement des indigènes au Diable à "l'inculturation" de l'Évangile (Manille 1982), via la religion populaire (Vatican II) et la théologie indienne de la conférence de l'épiscopat latinoaméricain (CELAM, Medellin, 1968). Dans ce cadre, les identités se sont formées à partir d'une gestion religieuse de l'espace social et de la permanence des représentations et des pratiques pré-hispaniques. Ce sont ces dernières qui, jusqu'à aujourd'hui, tendent à gérer la sphère privée et, par conséquent, les activités individuelles (rapports familiaux, gestion de la maladie et de la mort, systèmes symboliques liés à la production, à la terre, etc.). Ce système perdurera jusqu'aux années 1950. C'est alors qu'il entre en crise, celle-ci découlant de l'épuisement de ce qui en constituait jusqu'alors la logique organisatrice. Les raisons en sont multiples : croissance démographique à l'origine de l'exode rural, qui entraîna lui-même la fin des sociétés agraires; début d'ouverture à la logique du marché; perte d'influence de l'État ainsi que des partis politiques.

C'est dans le domaine des systèmes de parenté et d'autorité que l'éclatement des institutions traditionnelles fut particulièrement visible: le réseau familial n'apparaissant plus garant de l'accès à la subsistance, les individus se virent contraints de rechercher l'élargissement de leurs réseaux d'appartenance très au-delà de la seule structure familiale. Ce contexte fut inévitablement porteur d'une remise en cause radicale de l'influence et de l'emprise de l'Église catholique, tant celle-ci apparaissait étroitement liée à une structure traditionnelle dont elle avait constitué le pivot. La désarticulation de ces formes collectives classiques de représentation du monde déboucha sur l'entrée dans un univers fondamentalement caractérisé par la circulation, et, dès lors, c'est par la déterritorialisation que se manifesta un nouveau rapport à l'espace. Cette évolution entraîna des phénomènes d'ajustement, mettant en scène de nouvelles formes de gestion de la dérégulation du système traditionnel. L’Amérique latine étant par ailleurs un terrain de diffusion «naturelle» du modèle nord-américain, la situation fut marquée par un engagement massif de missionnaires et de financements. C'est sur cette base que le pentecôtisme connaîtra un développement exponentiel : en 1900, on estimait que le nombre d'évangélistes oscillait en Amérique latine entre 200 et 300000 ; ils étaient 21 millions en 1980, 46 en 1990, entre 80 et 90 en 2000. Autrement dit, près de $12 \%$ de la population latino-américaine serait évangélique. Au Guatemala ${ }^{5}$, certaines statistiques récentes établissent le nombre de protestants à près de $40 \%$ de la population. Au Chili, le taux serait de $25 \%$ et près de $22 \%$ au Salvador ${ }^{6}$. Suit le Brésil, où l'essor est des plus spectaculaires : 4 millions en 1960, 8 millions en 1970, 15 millions en 1980, 26 millions en 1990 et 35 en 2006 (soit $20 \%$ de la population). Au Nicaragua ${ }^{7}$, le 
pourcentage serait d'environ $16 \%$ de la population, au Panama de près de $15 \%$. L'amorce de ce développement est intervenue à l'aube du XXe siècle. Mais ce sont les années 1960 qui ont vu l'essor massif d'un tel courant. Se posant en "protestantisme de la modernité ", le pentecôtisme s'est adressé en priorité aux couches sociales les moins favorisées et au monde indien.

Dans les années 1970, une forme rénovée du pentecôtisme fait son apparition, d'où l'expression de néopentecôtisme pour désigner une mouvance qui, campant dorénavant sur un postmillénarisme (à la différence du pentecôtisme prémillénariste), va s'appliquer à définir des voies nouvelles pour "discipliner la société", notamment par le biais de la réarticulation des systèmes familiaux, s'adressant cette fois-ci prioritairement aux classes moyennes et aux bourgeoisies ${ }^{8}$.

Si les pentecôtistes considéraient que l'instauration du Royaume dépendait de la Seconde Venue du Christ et que, dans l'attente, il fallait s'éloigner du monde, gouverné par Satan, pour les néopentecôtistes, le Royaume de Dieu est déjà de ce monde et le chrétien doit en conséquence travailler à ce que, lors de la Seconde Venue du Christ, le monde lui soit déjà acquis. Il faut dès lors modifier la société : changer les constitutions et les lois afin qu'elles soient le reflet de la doctrine biblique. L'un des moyens pour y parvenir est d'occuper les espaces de pouvoir. Se voient légitimées d'autre part, en référence au calvinisme, la richesse et la santé comme résultat de la bénédiction divine. La majorité des fidèles appartenant à la classe moyenne et à la bourgeoisie, la participation politique et l'exercice du pouvoir sont justifiés? ${ }^{9}$. Les néopentecôtistes opposent à

5. De nombreuses sources fournissent des chiffres mais la Commission de formation du diocèse de Quetzaltenango, dans un document pour la «Rencontre de Réflexion diocésaine ", intitulé "Croissance des Églises évangéliques pour le Guatemala", Document de Référence n 4 du 6 juillet 1988, indiquait textuellement que «d'après les dernières données provenant de sources évangéliques, en juillet 1987, il y avait au Guatemala 2668810 personnes qui composaient la communauté évangélique, soit $31,6 \%$ de la population guatémaltèque, autrement dit, 31 guatémaltèques sur 100 seraient évangéliques». Ce document signale également que, à la même date, il y avait 3298 églises, soit 1 pour 906 habitants.

6. Pendant les années 1980, malgré la confrontation armée, les Églises évangéliques ont poursuivi leur développement : par exemple, la Mission centraméricaine, pendant toute la décennie, a connu un taux de croissance de 30\%. Les Assemblées de Dieu sont passées, entre 1980 et 1985 de 20000 à 80000 membres. Les conversions proviennent de tous les secteurs sociaux parmi lesquels les exguérilleros, les officiers de l'armée, les soldats (cf. Garry Parker, "Evangelicals Blossom Brightly Amid El Salvador's Wasteland of Violence ", in Christianity Today, mai 1981, 25 : 34).

7. Au Nicaragua, les évangéliques représentaient 3,2\% en 1979 mais, en 1983, ils atteignaient $12 \%$, bien qu’à Managua, la capitale, ils parviennent à 17\% (cf. P. Wagner, Spiritual Power and Church Growth, Altamonte Springs, Fl., Strang Communications, 1986).

8. Raisons des conversions : remariage, entrée dans l'univers de la circulation.

9. Voir Jesus Garcia Ruiz, «Pentecostales y Neo-pentecostales en Guatemala: ideología, espacio político y redes sociales", in J. Garcia-Ruiz, Cambio e identidad social: lo religioso en los procesos de recomposición en Guatemala, Estudios y Documentos, 14, Guatemala, Ediciones UnescoMuni-k'at, 1999 : 77. 
l'eschatologie défaitiste des pentecôtistes, une eschatologie de la victoire dont le fondement est la croyance dans la continuité historique entre le jour où l'Esprit Saint s'est manifesté aux apôtres et le jour du jugement dernier. Convaincus que Dieu leur a délégué Son pouvoir ${ }^{10}$, Son autorité ${ }^{11}$, $\mathrm{Sa}$ souveraineté ${ }^{12}$ et $\mathrm{Sa}$ domination ${ }^{13}$, ils mènent des stratégies de " conquête du monde et des nations" afin de les gagner au Royaume.

Le néopentecôtisme latino-américain a été profondément influencé par le protestantisme nord-américain. Cette influence s'est d'une part développée dans le mouvement évangélique; elle a, d'autre part, contribué à donner naissance à de nouvelles institutions. Au titre de la première logique, on mentionnera le Congrès mondial d'évangélisme qui a eu lieu à Berlin sous le patronage de Billy Graham (1966) et qui est à l'origine des Congrès latino-américains d'évangélisation (CLADE I et II). Au titre de la seconde, on évoquera El Movimiento del Crecimiento de Iglesias (Mouvement de croissance des églises) et le Réseau international de guerre spirituelle ${ }^{14}$. Une troisième logique interviendra, portée par les intérêts et les stratégies des groupes

10. Les néopentecôtistes utilisent en permanence cette terminologie: Power Evangelism (titre de l'œuvre de J. Wimber), fait référence à la proclamation de l'Évangile du Royaume de Dieu, qu'accompagnent des «signes, des prodiges et des merveilles ». En prêchant l'Évangile, des miracles et des "guérisons" se produisent, les démons sont jetés dehors. La notion de pouvoir dans le néopentecôtisme renvoie à la "totalité du Royaume de Dieu dans l'Univers", et ce, depuis trois perspectives: gouvernement de Dieu sur toutes les choses; règne spirituel de Dieu sur la vie du croyant sur la terre; royaume futur, c'est-à-dire que la terre, le ciel et les êtres seront réunis à la fin des temps. Autre sens possible: la "domination" particulière sur les êtres humains qui le reconnaissent de leur plein gré comme leur roi. Cela inclut son action dans l'histoire et la «souveraineté " générale sur l'univers.

11. L'autorité réside en Dieu. Une des interrogations de la Réforme se demandait si l'autorité de Dieu venait à l'être humain par le biais de l'Église (comme l'enseignait l'Église catholique) ou si elle venait par celui de la Bible (comme le disaient les Réformateurs). Plus récemment, la raison et l'institution spirituelle se sont transformées en autorité alternative. Dans certains cercles pentecôtistes et néopentecôtistes, le "dirigisme " de l'Esprit Saint est considéré comme expression d'autorité, ce qui les conduit à parler « d'autorité du ministère pour prêcher, enseigner, rendre le culte ou veiller sur les âmes, etc., qui vient de Dieu, à travers les Écritures et l'Église».

12. Les néopentecôtistes utilisent ce terme comme qualité de Dieu qui dénote Sa liberté face à tout type de restriction extérieure puisqu'Il est libre d'agir sans entrave. Par ailleurs, en tant que roi du Royaume, Il est souverain sur Lui-même et sur tous ceux qui L'ont accepté.

13. Rang, pouvoir et dignité du Christ « comme Seigneur des seigneurs, Roi des rois». Le terme renvoie à Sa domination sur toute la création (matérielle et spirituelle), sur toutes les circonstances (historiques et métahistoriques) et sur toutes les relations (divin-humains et humains-humains). La " confession" (au sens de déclaration de ce que l'on croit) fondamentale de tout croyant, c'est que "Jésus Christ est son Seigneur ", ce qui veut dire affirmer son "appartenance " au Christ par l'obéissance inconditionnelle à sa volonté.

14. Cette dimension de "guerre spirituelle" est devenue, ces dernières années, une composante centrale du comportement individuel: les pentecôtistes considèrent cette bataille comme la « défense " du Royaume de Dieu, les néopentecôtistes comme «l'attaque » contre le royaume de Satan. En effet, les pentecôtistes pensent qu'ils doivent rester "à l'écart du monde", autrement dit ils doivent se défendre contre l'empire du diable qui cherche leur perte, et se tenir prêts pour la Seconde Venue du Christ, tandis que les néopentecôtistes jugent qu'il faut livrer bataille contre le mal : il faut attaquer le diable pour participer à la restauration du Royaume et préparer le retour du Christ. 
sociaux dominants (classes moyennes, bourgeoisie, oligarchie et militaires), cherchant à se doter d'une idéologie légitimant leur domination et justifiant leur statut social. Le besoin de ces groupes de s'approprier des espaces sociaux et politiques constitue un véritable tournant en matière de formes historiques de représentation et de symbolisation du politique, induisant de nouvelles formes de participation et d'engagement ${ }^{15}$.

Le néopentecôtisme a généré une transformation tant de la doctrine (avec le développement de la "théologie de la prospérité et de la santé ») que des référents et des stratégies de conversion des groupes sociaux : c'est au niveau urbain et parmi les groupes des classes moyennes et hautes que leur action s'est développée. La "pentecôtisation ${ }^{16}$ de certains secteurs du protestantisme historique et du protestantisme de sanctification est également une réalité qu'il faut prendre en compte car les modalités d'interférence et d'action sociale s'en trouvent, par conséquent, profondément réorientées.

\section{Le pasteur : acteur local, acteur global}

Le néopentecôtisme apporte avec lui la transformation de la nature institutionnelle du religieux, du contenu idéologique de la doctrine, des systèmes d'administration et, surtout, du rôle d'un nouveau leadership individuel confinant au culte de la personnalité et articulé en réseaux. Les acteurs en sont des pasteurs locaux formés aux États-Unis qui mettent en place, à leur retour, des églises d'un nouveau type, s'adressant aux classes moyennes et à la bourgeoisie. Leurs premières réunions se sont tenues dans les salons de grands hôtels où les groupes concernés trouvaient des « conditions » adaptées aux exigences de leur statut social. Ces pasteurs pratiquent aujourd'hui de nouvelles formes de prosélytisme par "saturation », grâce à l'usage intensif des médias, à la multiplication des réunions, à des activités

15. J. Serrano Elias, La Participación del cristiano en la vida pública, Miami, Unilit, 1990 : 18.

16. Ce qu'on appelle le "dénominalisme " et les logiques institutionnelles des Églises traditionnelles sont en crise. Il est évident que, dans le contexte latino-américain, on peut de moins en moins parler de "principes baptistes" ou d'idées méthodistes: "De plus en plus ", dit P. Deiros, pasteur d'une Église baptiste néopentecôtiste de Buenos Aires, «l'épiderme dénominationnel devient perméable et nous influons et sommes influencés plus profondément par les autres, au sein de la famille évangélique ». J. Míguez Bonino ("Una crisis eclesiológica», in Boletín teológico, 25 septiembre, 1993: 186), pasteur méthodiste, résume efficacement la situation : «Ce à quoi nous assistons en Amérique latine, c'est à un regroupement de la communauté chrétienne. Cela peut vouloir dire, ou non, la fragmentation des structures ecclésiales. Mais, en tout cas, cela met entre parenthèses la question confessionnelle et fait ressortir différents types d'unité et de divergence. Il est pour le moins instructif que les nouvelles lignes de confession et de séparation ne soient pas aussi souvent débattues, mais que soient au contraire brandis le langage et les attitudes caractéristiques des temps polémiques confessionnels. Le fait même que tant de personnes et d'églises refusent d'envisager le problème sous un jour légitimement confessionnel, refusant aux "familles" dissidentes la dignité ecclésiastique est une preuve claire de la situation dans laquelle nous nous trouvons ». 
régulières et différenciées en fonction des classes d'âge, des groupes familiaux, etc. Cette activité déclenche des processus accélérés de conversion. Par ailleurs, la croissance du nombre de convertis s'accompagne de la constitution d'une masse critique financière grâce aux "dîmes" et aux dons qui permettent d'acheter des terrains et de construire des églises pouvant contenir plusieurs milliers de personnes. C'est ce que l'on observe à partir des années 1980. C'est le cas au Guatemala de la Fraternidad Cristiana, de la Iglesia El Shaddai, etc.

Une des caractéristiques majeures du néopentecôtisme réside dans le développement massif des Églises indépendantes. Cela est à l'origine de la fonction déterminante assignée au pasteur.

Dans la nouvelle logique d'Église indépendante, l'acteur central de la privatisation du religieux est de fait le pasteur. Il est tant le propriétaire de l'institution et des infrastructures que le producteur de sens et l'organisateur des relations. Ces Églises indépendantes, se pensant comme uniques, prétendent représenter la totalité du religieux et se donnent le monde comme lieu de leur action. Elles embrassent la société tout entière comme espace de mission. De là l'extraterritorialité et l'universalité qu'elles revendiquent. D'où l'importance attribuée au processus de conversion.

René Padilla, secrétaire exécutif de la Fraternité théologique latinoaméricaine, est équatorien et c'est à Quito que la Fraternité du 24 mai 1977 réunit trente dirigeants de neuf pays du continent pour «dresser le portrait du leader chrétien pour l'Amérique latine ${ }^{17}$. La réunion fut copatronnée par MAP América Latina, l'Overseas Ministry Studies Center (OMSC) de New Haven (Connecticut) et le Réseau chrétien de transformation intégrale. Il s'agit aussi d'un nouvel effort pour consolider des processus politiques à travers "l'élaboration du modèle de leaders dont les églises ont besoin dans la région ». Deux interventions avaient été préparées et considérées comme centrales: celle du chilien Oscar Pereira, membre fondateur de la Fraternité théologique latino-américaine, qui est intervenu sur " les modèles bibliques de leadership » et celle de Julio Carlos Cevalios sur "l'éducation théologique en Amérique latine ». Après avoir affirmé que le modèle de leadership doit être biblique et que le modèle idéal, c'est celui du Christ, les participants ont remarqué que dans la pratique, il faut proposer, avec des yeux latino-américains, des modèles éducatifs et d'urbanisation nouveaux, afin d'identifier les problèmes sociaux actuels et d'aider à transformer les structures internes de l'Église et «celles, extérieures, de la société qui limitent l'efficacité du Royaume de 
Dieu ». Un modèle spécifiquement latino-américain de leadership a été revendiqué, supposé prendre en compte «les grands changements et défis qui sont en train de se produire dans le monde ». Ce «modèle propre » de leader doit rompre avec les pratiques contemporaines que caractérisent des modèles importés, "pasteur-centriques ", imposés par les hiérarchies supérieures, hors contexte, caudilliste et qui ne sont plus adaptés à la réalité sociale. Conscients de leurs limites, les participants ont demandé à MAPA Amérique latine et à l'Overseas Ministry Studies Center (OMSC) qu'ils " jouent le rôle de stimulants » pour l'émergence de ce nouveau leadership.

Les Églises qui voient le jour dans la seconde moitié des années 1970 sont produites par le processus de globalisation, ce qui signifie qu'elles sont d'emblée porteuses de l'idéologie, des valeurs et des formes institutionnelles issues du management, des nouvelles techniques de communication et, plus généralement, de la valorisation de la logique du marché. Ces nouvelles modalités d'organisation et d'institutionnalisation sont caractérisées par l'autonomie hiérarchique, le nouveau rôle du pasteurleader-propriétaire et l'intégration dans des réseaux. Le néopentecôtisme transforme la nature institutionnelle du religieux, le contenu idéologique de la doctrine, les systèmes d'administration. Il met l'accent sur un nouveau leadership individuel confinant au culte de la personnalité et articulé en réseaux. Dans cette économie générale, le pasteur se pose en acteur global, universel, toute Église néopentecôtiste se devant d'être missionnaire. En d'autres termes, étant le Royaume de Dieu, elle doit s'impliquer dans son expansion et dans son rayonnement universels.

Pour ce faire le pasteur néopentecôtiste - autonome et indépendant cherche à se donner les moyens d'une efficacité globale. La structure de l'institution est simple et se fonde sur le principe de réduction d'effectifs propre à la logique de compétitivité. L'Église néopentecôtiste est dès lors et tout d'abord une affaire de famille: l'épouse est pasteur associé, certains fils également ; d'autres ont sous la responsabilité du pasteur la charge de certaines activités de l'Église (station ou programmes de radio ou de télévision, direction de l'orchestre de l'Église, etc.). En impliquant de la sorte sa famille, le pasteur prépare sa succession et la transmission de son patrimoine.

Par ailleurs, ces Églises néopentecôtistes recrutent des "pasteurs associés ", collaborateurs en charge d'activités concrètes (coordination des différents groupes de fidèles : enfants, jeunes, femmes, couples...) mais sans pouvoir de décision. Il s'agit de salariés susceptibles de le rester toute leur vie ou de partir créer leur propre église ou ministère du fait des compétences acquises au poste qu'ils occupent. Devenant à leur tour pasteurs-propriétaires, ils sortent du salariat. Le rôle et le statut du pasteur dans les Églises néopentecôtistes 
tranchent avec la situation qui peut prévaloir dans d'autres Églises. Ces Églises ont un faible degré de bureaucratisation. Le pasteur-propriétaire ne peut pas se permettre que le charisme se routinise, au sens où l'entendait Max Weber, en raison du contexte de forte concurrence dans lequel s'inscrivent ces Églises. Le pasteur-propriétaire incarne la dynamique charismatique de son Église ce qui implique une prise d'initiative permanente, sous peine de voir les fidèles abandonner l'Église. Cette logique fait que la dynamique de croissance se fonde sur des ruptures: plus il y a de ruptures, mieux cela vaut, car plus d'acteurs s'impliquent, plus de pasteurs émergent, plus d'institutions voient le jour.

Dans le développement des Églises, l'élément central de consolidation institutionnelle revient aux finances, la dîme et les offrandes des fidèles étant le moteur de cette croissance. En dépendent le pouvoir et l'influence du pasteur-propriétaire, la constitution d'un patrimoine et la visibilité sociale de l'institution. C'est évident au Guatemala: au terme des années 1990, près de trente Églises nationales s'engagent dans le développement de "méga-Églises», constructions de lieux de culte pouvant accueillir de très importants groupes de fidèles. L'Église «Casa de Dios », récemment inaugurée dans la capitale, peut ainsi contenir 4600 personnes assises. Une autre, dont la construction a coûté 4,5 millions de dollars US, peut accueillir 7000 personnes. Une troisième, la Mega-Frater, a une capacité de 15000 personnes et coûtera plus de 48 millions de $\$$. On retrouve le phénomène avec le développement des médias : la «Iglesia Familia de Dios ", en plus de la construction de son temple d'une capacité de 9000 personnes et qui a coûté 3,5 millions de $\$$, a également construit pour sa chaîne de télévision (Canal 27) un immeuble dont le coût dépasse les 3,5 millions de $\$$, sans compter les équipements techniques nécessaires à la production et à l'émission des programmes.

Le pasteur, pour dynamiser et donner de la visibilité à son institution, doit par ailleurs s'insérer dans des réseaux globaux. Ce recours aux réseaux devient une composante de la logique institutionnelle car c'est en même temps le lieu de légitimation de sa personne et de son institution. Par ailleurs, cette appartenance lui permet d'inclure de manière systématique des acteurs globaux prestigieux comme acteurs de sa communauté locale. En effet, il peut recruter pour des activités ponctuelles tel ou tel orateur de prestige qui viendra participer au culte de son Église ou à des activités plus ponctuelles (congrès...). Cette insertion dans les réseaux permet au pasteur local d'être à son tour invité et de devenir par là même acteur global. Fondés sur des relations d'interdépendances, ces réseaux transnationaux ont été développés par une nouvelle génération de leaders. 
Comme le signale David Cannistraci ${ }^{18}$, le réseau est là « essentiellement un regroupement d'églises autonomes et de ministères individuels qui s'unissent volontairement en une structure organisée. Le cadre de travail des relations humaines est suffisant pour faciliter l'interdépendance entre les membres du réseau de travail et sa supervision apostolique ». La terminologie utilisée dans cette description ("autonomes", " volontairement ", " relations ", " interdépendance ", "supervision ») met en évidence la véritable nature des réseaux observés sur le terrain. Ceuxci sont "translocaux" et transnationaux, ce qui signifie que leurs membres ne se retrouvent pas dans des espaces de compétence interne. Ils sont fondés sur des «relations» déterminées par l'interdépendance, ce qui implique une hiérarchie reconnue et acceptée volontairement; en d'autres termes, il s'agit de structures créées "par une nouvelle génération de leaders ${ }^{19}$, engagés agressivement dans des relations de pacte ${ }^{20}$. Avec le leader - souvent considéré comme un "apôtre " ${ }^{21}$ - les membres du réseau entretiennent des relations de confiance; d'où l'idée que les structures d'organisation sont fondées sur la confiance en une personne et non en des "équipes", des "comités " ou des "conseils». C’est cette "confiance" qui fait que le réseau croît ou s'éteint.

Le réseau est pensé comme une structure de service: «il sert et ne contrôle pas ". C'est en conséquence l'Église locale qui est porteuse de légitimité et non le réseau ; c'est donc l'ensemble des Églises locales qui conferent une légitimité au leader. Considérant que l'affiliation est volontaire et la structure mobile, les responsables du réseau prendraient le risque, s'ils tentaient de l'instrumentaliser, de se saborder eux-mêmes et d'être exclus du réseau, puisque ce sont les Églises locales qui font leur force. Cela signifie que le réseau doit toujours avoir la capacité d'offrir des services; cela signifie également que la prise d'initiative doit être permanente.

18. David Cannistraci, Apostles and the Emerging Apostolic Movement, Ventura, Regal, 1996 : 190.

19. Ce nouveau type de leadership a été décrit par John Echkardt, qui dirige sa propre institution, Crusaders Ministries, dont le siège est à Chicago.

20. Selon l'affirmation de Greg Dickow (fondateur et pasteur principal de l'Église «Life Changers International " de Barrington Hills [Illinois] qui coordonne le réseau du même nom), dans un polycopié intitulé "A New Breed of Leaders" (1998).

21. Il s'agit d'un des nombreux termes re-sémantisés par le mouvement évangélique. C'est l'un des "ministères" reconnus par le mouvement (à côté de celui de pasteur, de maittre, de prophète, d'évangéliste) et qui se réfere à une personne "porteuse de message ", qui " exerce une autorité en fixant et en développant une politique ». Dans plusieurs études, "l'apôtre " est décrit comme celui qui est capable "d'exercer un leadership général sur un certain nombre d'Églises avec une autorité exceptionnelle sur les questions spirituelles, autorité qui lui est reconnue spontanément». 
Dick Iverson ${ }^{22}$, fondateur de Ministries Fellowship International, aborde clairement ces nouvelles logiques d'articulation et d'institutionnalisation lorsqu'il affirme en présentant son institution :

"Nous nous sommes engagés à faire ce qu'il y avait à faire pour maintenir notre compagnonnage dans le réseau et éviter tout type de hiérarchie contrôlée. Nous ne nous opposons pas aux titres ni à ceux qui les ont [...] pourtant notre conviction personnelle est qu'un siège central ne doit pas dicter la politique de nos Églises locales. Chaque Église doit être autonome; les pasteurs peuvent jouir de la sécurité d'une responsabilité significative mais également voir leur leadership vérifié et pesé.»

Le réseau est donc pensé comme une articulation des Églises locales, sans hiérarchie et autonomes, ce qui reste, pour de nombreux responsables, la meilleure méthode contre la "routinisation générale du charisme». Le but est d'arriver à un réseau de réseaux, où les Églises et les institutions locales auraient la possibilité de s'articuler en fonction de leur sensibilité mais aussi en fonction de leur évolution dans le temps; pouvoir entrer et sortir librement en reprenant constamment l'initiative: à un moment donné, l'Église locale sera intéressée par les thématiques et les dynamiques de tel ou tel réseau, puis son évolution pourra l'amener à s'intéresser à d'autres thèmes traités dans un autre réseau ; c'est alors ce nouveau réseau qui fournira une valeur ajoutée au développement de l'institution. Le réseau réactive continuellement les dynamiques et garantit la privatisation institutionnelle du religieux puisque le leader-pasteur-propriétaire acquiert un rôle central : il est le garant, d'une part, de la présence de son institution dans le réseau et, d'autre part, de sa mobilité et de son adaptabilité aux changements.

Le développement du néopentecôtisme constitue certes un lieu privilégié d'observation des nouvelles valeurs de sociétés travaillées par un mouvement avec lequel des affinités lui sont prêtées. Il représente également un indicateur des modalités d'établissement, par ces sociétés, d'un rapport au pluralisme, dont il apparaît d'ailleurs comme l'une des composantes. Il est, partout, tenté de passer du statut d'acteur religieux à celui d'acteur social, économique et, surtout, politique, à tel point qu'on peut affirmer que s'il est acteur religieux, social et politique c'est parce qu'il est, avant tout, acteur économique. La constante prise d'initiative par des acteurs qui se considèrent comme "élus" reformule par ailleurs les logiques de légitimité. La privatisation des croyances entraîne celle des Ventura, Regal, 1998 : 176. 
institutions, de leur dimension financière, de leur capacité à articuler des modes de fonctionnement communautaires, de la légitimité de leur appartenance. En ce sens, les logiques historiques qui régulaient les rapports entre État et société tendent à se transformer. Ce qui signifie, entre autres, que, sous couvert de rétablissement d'une "discipline», ce qui est visé ressortit plutôt à une tentative pour instaurer un " contrôle ». Mais le sens général des évolutions dans lesquelles s’inscrivent tant la progression des néopentecôtistes que l'usage qu'en font les acteurs, demeure frappé au coin d'une incertitude fondamentale. On voit bien comment, partant de l'individuation contemporaine et de la pleine légitimité sociale reconnue à celle-ci, des entrepreneurs religieux promeuvent la privatisation du religieux et constituent celle-ci en un vecteur performant d'une recommunautarisation pensée comme instrument, à terme, d'une néopentecôtisation de l'espace public. Il n'en demeure pas moins que ce qui est conçu par ces entrepreneurs comme le point d'arrivée de l'ensemble du processus pourrait bien n'être, pour ceux qui s'y trouvent impliqués, qu'une étape (et un moyen) d'un processus de plus grande ampleur, dans lequel la néopentecôtisation ne serait guère qu'un moment dans la construction d'individus s'éprouvant comme tels dans le mouvement. Et, dans cette perspective, l'émergence récente au Guatemala, par exemple, d'acteurs politiques directs - avec un parti propre - va dans ce sens.

Dans la modernité, l'État était un facteur central dans le processus d'individuation (formation civique, éducation); dans la globalisation, l'État est en partie dépossédé de ce rôle de matrice de l'individuation. D'autres institutions tendent à se substituer à lui (cas de l'éducation catholique à partir des années 1960 : développement d'écoles et d'universités pour la formation des élites; cas de la mouvance évangélique à partir des années 1980), en plus du secteur privé (qui se saisit de l'éducation comme d'un secteur participant comme les autres de l'économie avec une logique de l'investissement et du profit). La religion se vit dans la modernité comme contraire à cette modernité. Dans la globalisation, certains des éléments constitutifs du paysage religieux se vivent en revanche comme en phase parfaite, et non plus ayant à s'adapter mais susceptibles de produire de la médiation, d'être des acteurs du global.

Centre national de la recherche scientifique, CEIFR jesus.garcia-ruiz@wanadoo.fr

MOTS CLÉS/KEYWORDS : anthropologie religieuse/religious anthropology-millénarismes/millenarianisms - (néo)pentecôtisme/ (neo)pentecostalism - Amérique latine/South America - Guatemala. 